\title{
MACROINVERTEBRADOS BENTÓNICOS COMO INDICADORES DE INTEGRIDAD ECOLÓGICA DEL SIS- TEMA FLUVIAL RÍO DULCE (SANTIAGO DEL ESTERO)
}

Marta Elisabeth Leiva

martaleiva@gmail.com

Doctorado en Ciencias Biológicas

Directora: Dra. Mercedes Marchese (INALI, UNL-CONICET)

Lugar de realización: Instituto de Protección Vegetal, Facultad de Ciencias Forestales, Universidad Nacional de Santiago del Estero (INPROVE, FCF, UNSE)

Fecha de la defensa: 15 de marzo de 2019

\section{RESUMEN}

El uso de los macroinvertebrados bentónicos como indicadores del estado ecológico de los ríos constituye un área de conocimiento en continuo crecimiento. Uno de los factores que influyen para que estas investigaciones cobren mayor impulso es el hecho de que los ecosistemas fluviales están cada vez bajo mayor presión, debido a los variados servicios que proveen a las actividades humanas. La misma razón hace necesario ampliar el conocimiento ecológico necesario para que su aprovechamiento no comprometa la conservación de sus funciones ecosistémicas. El Río Dulce, en Santiago del Estero, Argentina, es afectado por múltiples presiones antrópicas, principalmente en relación a la regulación de caudales, la pérdida de los ecosistemas de ribera y la contaminación. Al presente, no se ha desarrollado aún una base de conocimiento ecológico que permita proponer herramientas para el manejo y monitoreo de este sistema fluvial. En este trabajo de tesis se propuso un estudio sobre la ecología del Río Dulce enfocado en los ensambles de macroinvertebrados bentónicos, con el objetivo de proponer herramientas para su utilización como indicadores de integridad ecológica. Para ello se plantearon muestreos en sitios localizados sobre el gradiente longitudinal del río en periodos de aguas bajas y de post-crecida, y se analizó la calidad de aguas, las características del hábitat, variables hidrológicas y morfológicas, y se analizaron las condiciones de las riberas, con el objetivo de lograr una evaluación integral del sistema fluvial. Como resultados principales, respecto de la calidad de agua se evidencia sobre el gradiente longitudinal un impacto antrópico relacionado a las fuentes de contaminación urbana. La granulometría del lecho con alta proporción de arenas y materiales gruesos, en conjunto con la velocidad de la corriente, resultan características particulares del sistema fluvial, que influyen sobre la composición y estructura de los ensambles bentónicos. Se registraron 100 taxa identificados a diferentes niveles taxonómicos, de los cuales la familia Chironomidae de Insecta y la Clase Oligochaeta fueron dominantes. Los organismos colectores-recolectores fueron el grupo funcional trófico predominante. Se observó una alta diversidad $\beta$ relacionada principalmente al reemplazo taxonómico entre los sitios, así como una 
importante variabilidad temporal relacionada al pulso de crecida estacional. Se detectó una fuerte influencia de la alteración hidrológica, dada por la sustracción de caudales hacia el sistema de riego. Las riberas del Río Dulce muestran moderados a fuertes niveles de impacto, con degradación del bosque de ribera, y pérdida irreversible de la vegetación y morfología natural en las áreas urbanizadas. Los ensambles de macroinvertebrados bentónicos registraron el gradiente de impacto sobre la calidad del agua, aunque estuvieron muy influenciados por las características físicas del hábitat. Esto plantea la necesidad de ajustar aspectos metodológicos relacionados a la selección de los sitios con el objetivo de un adecuado biomonitoreo de calidad de aguas. Los quironómidos y oligoquetos mostraron valor indicador a nivel de géneros y especies, por lo tanto, se plantea la necesidad de profundizar los estudios a fin de aprovechar el potencial indicador de niveles de resolución taxonómica más finos, respecto de estos taxa. Se concluye que en el tramo del Río Dulce estudiado se logra una diferenciación de los sitios en base a los índices bióticos y los impactos identificados, separando a aquellos sitios que muestran una mejor calidad ecológica de aquellos con menores valores de calidad ambiental. Se concluye que es factible utilizar a los ensambles de macroinvertebrados bentónicos como indicadores de integridad ecológica en el Río Dulce, porque hay una estructura de ensambles diversa y asociada a diferentes características tanto de calidad de aguas como hidrológicas, morfológicas y en relación a los impactos antrópicos que se pudieron identificar en el sistema fluvial.

\section{ABSTRACT}

\section{Benthic macroinvertebrates as ecological integrity indicators in the Dulce river fluvial system (Santiago del Estero)}

By As several rivers around the world, Dulce River, in Santiago del Estero province, Argentina, is under multiple anthropogenic pressures, like flow regulation, riverine degradation, and pollution. There is not an ecological baseline in order to propose tools for biomonitoring of this fluvial system. The present thesis was conducted as an ecological approach, focused on benthic macroinvertebrates assemblages. The main goal was to obtain information for the development of integrity ecological indicators. It was evaluated biota composition and structure, water quality, fluvial habitat features, hydrological and morphological variables, and riverine landscape condition. It was recorded one hundred taxa at different taxonomic levels. Chironomidae and Oligochaeta were dominants. Collector-gatherers were the most abundant functional feeding group. High $\beta$ diversity was related to turnover between sites. Water quality shows anthropogenic impacts regarding urban pollution sources. Granulometry, current velocity, organic matter, and water quality constitutes drivers for benthic assemblages. Density and structure exhibit high temporal variability related to the seasonal spate. It was detected a strong hydrological 
alteration, induced by water abstraction. Riverine landscapes shown riparian forest degradation and loss of morphological features in urban areas. Benthic macroinvertebrates assemblages reflected the gradient of water quality and anthropogenic impacts. The conclusion is that is possible to use benthic macroinvertebrates as ecological integrity indicators in the Dulce River, because there is a diverse assemblage structure, associated with water quality but also with hydrological and morphological features, and regarded to anthropogenic impacts identifies in the fluvial system. 